\title{
Anarchic Yukawas and top partial compositeness: the flavour of a successful marriage
}

\author{
A. Parolini* \\ KAIST and IBS CAPP \\ E-mail: parolini@kaist.ac.kr
}

\begin{abstract}
We report here the content of a talk given by the author to Planck 2015, based on [1] and concerning flavour aspects of the generation of Standard Model fermion masses in models of pseudo Nambu Goldstone Boson composite Higgs. Indeed the top quark can be naturally singled out from other fermions due to its large mass, of the order of the electroweak scale: we consider a new class of flavour models, where the top quark obtains its mass via partial compositeness, while the lighter fermions acquire their masses by a deformation generated at a high flavour scale. One interesting feature of such scenario is that it can avoid all the flavour constraints without the need of flavour symmetries. We show that both flavour conserving and violating constraints can be satisfied without invoking any flavour symmetry for the up-type sector, in the case of the minimal $\mathrm{SO}(5) / \mathrm{SO}(4)$ coset with top partners in the four-plet and singlet of $\mathrm{SO}(4)$. In the down-type sector, some degree of alignment is required if all down-type quarks are elementary. We show that taking the bottom quark partially composite provides a dynamical explanation for the hierarchy causing this alignment.
\end{abstract}

18th International Conference From the Planck Scale to the Electroweak Scale 25-29 May 2015

Ioannina, Greece

* Speaker. 


\section{Introduction}

One very attractive idea to cope with the Standard Model (SM) hierarchy problem is to replace the Higgs doublet with a whole strong interacting sector. It is possible to generate a Higgs-like state among the pseudo-Nambu Goldstone Bosons (pNGB), like pions, of the spontaneous symmetry breaking of a global symmetry. In addition, for the top, the idea of partial compositeness [2] has been put forward: the existence of fermionic spin-1/2 states with the same quantum numbers of the top is postulated. They couple to the source of electroweak symmetry breaking (EWSB), being part of the composite sector, while a linear coupling with the elementary fermions allows for a propagation of the symmetry breaking to the quark sector. The advantage over a direct coupling of a top bilinear to the composite sector $[3,4]$ is the absence of dangerous four-fermion operators that may mediate large flavour changing effects [5].

Composite Higgs Models (CHM) with a quasi-conformal behaviour have been associated to models on a warped extra dimensional background [6, 7, 8], thanks to the AdS/CFT correspondence [9]. Following the success of such models [10,11], intensive studies of effective models of pNGB CHM have sprouted $([12,13]$ for reviews). Partial compositeness is considered a key ingredient, because flavour structures explaining the fermion hierarchies [14] and the absence of flavour changing neutral currents (FCNC) are automatically in place in the extra dimensional construction. On the other hand also examples of four dimensional dynamics can be found [15, 16, 17, 18, 19, 20, 21].

From the low energy effective theory point of view, pNGB CHM typically need flavour symmetries [22], as the flavour scale is set by the $\mathrm{TeV}$ scale. A comment is in order: in partial compositeness for all the quarks framework the flavour puzzle in new physics is mostly solved, a feature which makes partial compositeness very appealing. This is manifested in 5D models, where a Randall-Sundrum (RS) Glashow-Iliopoulos-Maiani mechanism is built-in [23], representing a major achievement. However, there is a residual tension left from $\varepsilon_{K}$ and electric dipole moments (EDMs), which requires the compositeness scale to be still as high as $O(10) \mathrm{TeV}$ [22, 24]. To avoid this little hierarchy, some kind of flavour symmetries (e.g. horizontal symmetries, alignments, minimal flavour violation with $\mathrm{SU}(3)$ or $\mathrm{U}(2)$, etc.) are still required, both in 5D holographic models [25, 26, 27, 28, 29, 30] and 4D composite Higgs models [22, 31, 32, 33].

In [1] a scenario of pNGB composite Higgs has been considered where partial compositeness is present for the top quark only ${ }^{1}$, while the masses of light quarks and leptons are generated by the more traditional direct coupling to the dynamics, as in technicolour theories [3, 4], in particular in conformal technicolour [34]. One reason for this choice is the difficulty in defining a simple underlying dynamics. Another issue relates to the fact that it might be debatable the assumption of a flavour symmetry in the strong sector given that it couples differently to the three SM generations. The case where both partial compositeness and direct couplings are present has some advantages: the top is uniquely defined as the combination of elementary fermions that couples to the fermionic partners, thus one can consider a truly flavour blind underlying dynamics. Furthermore, as the large top mass is generated by the partial compositeness, the direct couplings can be suppressed by a larger energy scale (just enough to generate the bottom, charm and tau masses) so that the sector responsible for the generation of such terms can be pushed to scales which are safe with respect to flavour violating effects, namely $O\left(10^{5}\right) \mathrm{TeV}$. These benefits would however only hold

\footnotetext{
${ }^{1}$ Potentially also the bottom quark can be partially composite.
} 
if no additional flavour symmetries are needed. We will therefore analyse the effects of bounds from both flavour-violating and flavour-conserving processes on a scenario where the light fermion flavour structure is anarchic at the high flavour scale. We anticipate that two different small ratios play an important role: $m_{c} / m_{t}$ and $m_{t} / M_{*}$, where $M_{*} \sim 1 \mathrm{TeV}$ indicates the scale of new physics. While the smallness of the latter is related to the smallness of $v / f, f \sim 1 \mathrm{TeV}$ being the decay constant associated to the coset of the breaking, the former suppression is truly a result of the interplay of two different mass sources. The solution of the hierarchy problem needs to single out a combination of quarks and make it heavier, causing the emergence of an approximate $\mathrm{U}(2)$ symmetry in the up sector. A similar attitude has been put forward in MSSM-like extensions of the SM [32, 35] and more recently in two Higgs doublets models, both in the leptonic [36] and in the quark sector [37]. We finally stress that models with heavy vector-like quarks, with or without a composite Higgs, might share the same properties as our framework.

\section{A composite Higgs model with additional Yukawa interactions}

A strong dynamics sector appears above a scale $f$, for instance deriving from the condensation of a "hyper-colour" gauge group at a higher scale $\Lambda_{H C}$ : following naive arguments, we fix $4 \pi f \simeq \Lambda_{H C}$. Between these two scales, all the heavy resonances appear, including top partners, while above the scale $\Lambda_{H C}$ we postulate the existence of a different dynamics that generates direct couplings of the elementary fermions (both quarks and leptons) to the strong sector at a high scale. In order to sufficiently suppress FCNC generated at the same scale, we will require that $\Lambda_{U V} \gtrsim 10^{5}$ $\mathrm{TeV}$ without assuming CP. $f$ needs to be at the TeV scale for naturalness. We also postulate the existence of a gap between $f$ and the electroweak (EW) scale $v$, so that the masses of the pNGBs are at a scale $v$, compatible with the measured value of the Higgs mass and electroweak precision tests (EWPT) are passed, at the price of a small, $O\left(v^{2} / f^{2}\right)$, fine tuning.

\subsection{Particle content and Lagrangian}

We restrict ourselves to the pNGB CHM with the minimal coset preserving a custodial symmetry, namely $\mathrm{SO}(5) / \mathrm{SO}(4)$ [11], and we embed the left-handed quarks $q_{L}=\left(t_{L}, b_{L}\right)^{T}$ and the right-handed one $t_{R}$ in spurions transforming in the fundamental 5 of $\mathrm{SO}(5)$ [38, 39]: this set-up is also known as $\mathrm{MCHM}_{5}$. We also assign to the elementary spurions a charge $\pm 2 / 3$ under an additional local $\mathrm{U}(1)_{X}$. The Higgs doublet, whose components are identified with the pNGBs, always appears through a unitary matrix $U$. We define $\Sigma=U \cdot(00001)^{t}$, transforming as a $\mathbf{5}$ of $\mathrm{SO}(5)$. We also define $\varepsilon=\langle h\rangle / f, s_{\varepsilon}=\sin \varepsilon$ and $c_{\varepsilon}=\cos \varepsilon$. The EW scale is set by $v=s_{\varepsilon} f \simeq 246 \mathrm{GeV}$, and we focus on values $s_{\varepsilon}^{2} \sim 0.1$ which implies $f \simeq 800 \mathrm{GeV}$, as set by EWPT [40].

We expect the composite sector to contain many spin-1/2 fermionic resonances, we include here a four-plet $Q$ and a singlet $\tilde{T}$ of $\operatorname{SO}(4), \psi=(Q, \tilde{T})^{t}$, The most general Lagrangian is then

$$
\begin{aligned}
\mathscr{L}_{\text {comp }} & =i \bar{Q}_{L, R}(\not D+\not L) Q_{L, R}+i \bar{T}_{L, R} \not D \tilde{T}_{L, R}-M_{4} \bar{Q} Q-M_{1} \overline{\tilde{T}} \tilde{T}+i c_{L, R} \bar{Q}_{L, R}^{i} \gamma^{\mu} d_{\mu}^{i} \tilde{T}_{L, R}+\text { h.c. } \\
-\mathscr{L}_{\text {mix }} & =y_{L 4,1} f \bar{q}_{3 L}^{5} U \psi_{R}+y_{R 4,1} f \bar{t}_{R}^{5} U \psi_{L}+\text { h.c. }
\end{aligned}
$$

where $E_{\mu}$ and $d_{\mu}$ denote the CCWZ Cartan-Maurer one-forms (c.f., e.g., [41]). The terms in $\mathscr{L}_{\text {mix }}$ are responsible for top partial compositeness: together with the gauging of $\mathrm{SU}(2)_{L} \times \mathrm{U}(1)_{X}$ they 
lift the Higgs and radiatively induce EWSB. We fix the masses of the heavy partners to be around 1 $\mathrm{TeV}$, and above the currents experimental bounds, roughly $650-800 \mathrm{GeV}[42,43,44]$. Multi TeV resonances would be the naive expectation but lighter masses are preferred by the light Higgs mass $[45,46,47]$ and therefore we assume this is the case, loosely identifying $M_{*} \sim M_{4} \sim M_{1} \sim \mid M_{1}-$ $M_{4} \mid \sim f$. Also we explicitly checked that a fully composite $t_{R}$ does not change our conclusions.

In addition we assume the presence of direct Yukawa interactions of all fermions, quarks and leptons, generated at a scale $\Lambda_{U V}>\Lambda_{H C}$ : they appear in the effective Lagrangian as couplings between pairs of SM elementary fermions and composite operators. This mixed possibility, top partial compositeness and additional deformations for the other quarks, has been recently considered in $[21,20,48]$. We loose the nice features of partial compositeness but we can study microscopical models in realistic situations and still account for a single separation of scale, between the top and all the other quarks. We then complement the Lagrangian with the following interactions

$$
\mathscr{L}_{Y}=\bar{q}_{L, \alpha} \lambda_{\alpha, \beta}^{u} u_{R, \beta} \mathscr{O}_{u}+\overline{\tilde{q}}_{L, \alpha} \lambda_{\alpha, \beta}^{d} d_{R \beta} \mathscr{O}_{d}+\text { h.c. }
$$

generated at the scale $\Lambda_{U V}$, where $\alpha$ and $\beta$ are indices over the 3 SM generations, and $\mathscr{O}_{u, d}$ are operators of the new dynamics. As these terms are generated independently on the partial compositeness, their embedding in $\mathrm{SO}(5)$ is free. We choose the same embedding as of the top: the fields $q_{L}$ and $u_{R}$ are thus a generalization of the elementary spurions to include three families, and $\tilde{q}_{L}$ and $d_{R}$ are defined in a similar way, with $\mathrm{U}(1)_{X}$ charge $\pm 1 / 3 . \mathscr{O}_{u, d}$ are composite operators in a non trivial representation of the broken $\mathrm{SO}(5)$ interpolating at low energy the Higgs doublet. If these operators are in a representation contained in $\mathbf{5} \times \mathbf{5}$ of $\mathrm{SO}(5)$, at low energy we obtain the following

$$
\mathscr{L}_{Y}=\sqrt{2}\left[\left(\bar{q}_{L}^{5} \Sigma\right) m_{\mathrm{UV}}^{u}\left(\Sigma^{T} u_{R}^{5}\right)+\left(\overline{\tilde{q}}_{L}^{5} \Sigma\right) m_{\mathrm{UV}}^{d}\left(\Sigma^{T} d_{R}^{5}\right)\right]=\frac{s_{2 \theta}}{2}\left[\bar{u}_{L} m_{\mathrm{UV}}^{u} u_{R}+\bar{d}_{L} m_{\mathrm{UV}}^{d} d_{R}\right]
$$

where $m_{\mathrm{UV}}^{u, d} \propto \lambda^{u, d}$ such that $s_{2 \varepsilon} m_{\mathrm{UV}}^{u, d} \sim O(1) \mathrm{GeV}$ to correctly reproduce the charm and bottom masses. The way $U$ appears is fixed by the representation of the operators $\mathscr{O}_{u, d}$ : our choice gives the same dependence obtained for the top from partial compositeness ${ }^{2}$. If the composite sector is fundamentally a gauge theory of strongly interacting fermions $\Psi_{i}$ one can secretly imagine the operators $\mathscr{O}_{u, d}$ as $\Psi_{i} \Psi_{j}$ bilinears. This reminds us of conformal technicolour theories; in the following, we will dub these terms UV, independently on their physical origin.

These operators have a small impact on the Higgs potential: they do not play a significant role for what concerns naturalness. Finally we stress that $\lambda^{u, d}$ are $3 \times 3$ generic matrices in generation space with rank 3. This mass term of order $O(1 \mathrm{GeV})$ in the up sector causes a misalignment between the physical top and the top defined as the partially composite quark.

\subsection{The structure of the model}

The fermionic field content defined above can be split into up and down sectors as $\xi_{\uparrow}=$

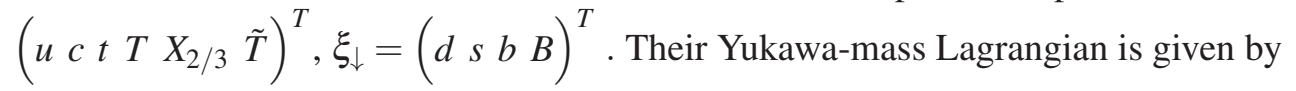

$$
-\mathscr{L}_{\text {yuk }- \text { mass }}=\bar{\xi}_{\uparrow L}\left[M_{\text {up }}+Y_{\text {up }} h+\cdots\right] \xi_{\uparrow R}+\bar{\xi}_{\downarrow L}\left[M_{\text {down }}+Y_{\text {down }} h+\cdots\right] \xi_{\downarrow R}+\text { h.c. }
$$

\footnotetext{
${ }^{2}$ A simpler choice could be to have composite operators in the $\mathbf{5}$ and embed right-handed quarks in $\mathrm{SO}(5)$ singlets: as a result we would have a different Higgs dependence in the effective Lagrangian, namely a single $\Sigma$ would appear. This choice would not significantly affect our analysis.
} 
We first define a change of basis to recover the mass eigenstates. Since this cannot be done exactly we use $s_{2 \varepsilon}$ as an expansion parameter: in the elementary sector a general $3 \times 3$ matrix is thus a perturbation to the null matrix. In other words, the unitary matrices that we shall find do not completely diagonalize the $6 \times 6$ (or $4 \times 4$ ) matrix, but only block diagonalizes it. Nevertheless, this is enough since in this new basis the heavy eigenstates are diagonal and they can be safely integrated out at tree level. For the up sector we get, up to $O\left(s_{2 \varepsilon}^{3}\right)$,

$$
U_{u L}^{\dagger} M_{\mathrm{up}} U_{u R} \simeq\left(\begin{array}{cc}
m_{U} & 0 \\
0 & D_{M}
\end{array}\right), m_{U} \simeq \frac{s_{2 \varepsilon}}{2} m_{\mathrm{UV}}^{u}+m_{t} \Pi, \Pi=\left(\begin{array}{ccc}
0 & 0 & 0 \\
0 & 0 & 0 \\
0 & 0 & 1
\end{array}\right), D_{M} \simeq \operatorname{diag}\left(M_{T}, M_{4}, M_{\tilde{T}}\right),
$$

where $m_{t}$ is the contribution to the top mass from partial compositeness (so that $s_{2 \varepsilon} m_{\mathrm{UV}} \sim m_{c} \ll$ $\left.m_{t}\right)$. We also define $s_{\phi L}=y_{L 4} f / M_{T}, s_{\phi R}=y_{R 1} f / M_{\tilde{T}}$. The Yukawa couplings are brought to a non block-diagonal form. The Yukawa matrix for the light quark sector is now given by

$$
y_{u} \simeq \frac{m_{U}}{f s_{2 \varepsilon} / 2}\left(1-\frac{1}{2} s_{2 \varepsilon}^{2}\right)+B_{u}, \text { where } B_{u} \sim \frac{\Sigma_{u}}{M_{*}^{2}}, \Sigma_{u} \sim\left(\begin{array}{ccc}
m_{c}^{2} & m_{c}^{2} & m_{c} m_{t} \\
m_{c}^{2} & m_{c}^{2} & m_{c} m_{t} \\
m_{c} m_{t} & m_{c} m_{t} & m_{t}^{2}
\end{array}\right) .
$$

For the down sector we obtain

$$
U_{d L}^{\dagger} M_{\mathrm{down}} U_{d R} \simeq\left(\begin{array}{cc}
m_{D} & 0 \\
0 & M_{T}
\end{array}\right), \quad m_{D} \simeq \frac{s_{2 \varepsilon}}{2} m_{\mathrm{UV}}^{d} .
$$

The Yukawa coupling in the down sector is decomposed in aligned and non aligned parts as

$$
y_{d} \simeq \frac{m_{D}}{f s_{2 \varepsilon} / 2}\left(1-\frac{s_{2 \varepsilon}^{2}}{2}\right)+B_{d}, B_{d} \sim \frac{m_{b} \Sigma_{d}}{\varepsilon M_{*}^{3}}, \quad \text { where } \quad \Sigma_{d} \sim \varepsilon^{2}\left(m_{\mathrm{UV}}^{d}\right)^{2} .
$$

The interaction Lagrangian of the EW gauge currents is

$$
\mathscr{L}_{\text {gauge }}=Z_{\mu} \bar{\xi}_{\uparrow L, R} \gamma^{\mu} A_{N C}^{t L, R} \xi_{\uparrow L, R}+Z_{\mu} \bar{\xi}_{\downarrow L, R} \gamma^{\mu} A_{N C}^{b L, R} \xi_{\downarrow L, R}+W_{\mu}^{+} \bar{\xi}_{\uparrow L, R} \gamma^{\mu} A_{C C}^{L, R} \xi_{\downarrow L, R}+\text { h.c. }
$$

Applying the transformations $U_{u L, u R}$ and $U_{d L, d R}$ to the EW gauge currents we obtain deviations as:

$$
\begin{aligned}
\left.\delta A_{N C}^{t L}\right|_{3 \times 3} & \simeq-\left.\delta A_{N C}^{t R}\right|_{3 \times 3} \simeq \frac{g}{c_{W}} \frac{\Sigma_{u}}{M_{*}^{2}},\left.\delta A_{N C}^{b L}\right|_{3 \times 3}=0,\left(\left.\delta A_{N C}^{b R}\right|_{3 \times 3}\right)_{i j} \simeq-\frac{g}{2 c_{W}} \frac{\Sigma_{d}}{M_{*}^{2}} ; \\
\left(\delta A_{C C}^{L}\right) & \simeq-\frac{g}{\sqrt{2}} \frac{\Sigma_{u}}{M_{*}^{2}}, \quad\left(\delta A_{C C}^{R}\right) \simeq-\frac{g}{\sqrt{2} M_{*}^{2}} m_{b} m_{t} \Sigma_{u} .
\end{aligned}
$$

In order to go from this basis to the "true" mass basis we need to perform unitary transformations acting on the light sector only, $m_{U}=V_{u L} M_{U} V_{u R}^{\dagger}, m_{D}=V_{d L} M_{D} V_{d R}^{\dagger}$ where $M_{U}=\operatorname{diag}\left(m_{u}, m_{c}, m_{t}\right)$ and $M_{D}=\operatorname{diag}\left(m_{d}, m_{s}, m_{b}\right)$ are the masses of the six quarks. Given the fact that $O\left(s_{2 \varepsilon} m_{\mathrm{UV}}\right) \sim O\left(m_{c}\right)$, the matrix $m_{U}$ contains a strong hierarchy due to the $\{3,3\}$ entry. Therefore $V_{u L, R}$ have the form

$$
V_{u L, R} \sim\left(\begin{array}{ccc}
O(1) & O(1) & O\left(\frac{m_{c}}{m_{t}}\right) \\
O(1) & O(1) & O\left(\frac{m_{c}}{m_{t}}\right) \\
O\left(\frac{m_{c}}{m_{t}}\right) & O\left(\frac{m_{c}}{m_{t}}\right) & 1
\end{array}\right)
$$

such that $V_{u L}^{\dagger} \Sigma_{u} V_{u R} \sim \Sigma_{u}$. In the down sector there is no a priori hierarchy between the mass matrix entries, except from the fact that is has to accommodate the down-type quark spectrum. 


\section{Confronting the model with data}

In this section we confront our model with the present constraints coming from flavour conserving/violating processes and also comment on precision data, non linearities and neutron EDM. All these effects may be induced solely by the mixing effects due to top partial compositeness and direct Yukawa couplings, thus appearing as flavour-violating couplings of the $Z, W$ and Higgs, they may be induced by heavy resonances appearing at the compositeness scale or they can be the result of the dynamics that generates elementary Yukawa couplings at the scale $\Lambda_{U V}$.

For what concerns third family couplings to $Z$ and $W$ bosons they get deviations as in pNGB CHM with partial compositeness $[39,49,50,48]$ and the model can be reconciled with experimental measurements for reasonable values of parameters. Flavour violating top couplings are present but induced $\mathscr{B}(t \rightarrow c h, t \rightarrow Z q)$ are orders of magnitude below the limits set by ATLAS [51] and CMS [52, 53]. Flavour violating $h$ and $Z$ couplings involving light families are controlled by $\Sigma_{u}$ and $\Sigma_{d}$, and they are tamed in the up sector. We included the effects of heavy, $O(1) \mathrm{TeV}$ masses, resonances coupled to top partners on $\Delta F=2$ processes. Finally a scale of generation of flavour $\Lambda_{U V} \simeq 10^{5} \mathrm{TeV}$ is consistent with the size of charm and bottom masses if we assume a conformal behavior of the theory between $\Lambda_{U V}$ and $\Lambda_{H C} \simeq 10 \mathrm{TeV}$ and anomalous dimensions for the operator $\mathscr{O}_{u, d}$ close to one $[54,55]$, together with the requirement of perturbativity of the couplings $\lambda^{u, d}\left(\Lambda_{U V}\right)$ : therefore the operators $(\bar{q} q)(\bar{q} q) \Lambda_{U V}^{-2}$ do not induce detectable effects [56, 57].

The combined analysis gives:

$$
\begin{aligned}
\mathrm{Z} \text { boson FCNCs } \Rightarrow & \left|V_{d L 33}^{*} V_{d L 13}\right|<10^{-1}, \quad\left|V_{d L 33}^{*} V_{d L 23}\right|<10^{-1 / 2}, \quad\left|V_{d L 13}^{*} V_{d L 23}\right|<10^{-5 / 2}, \\
\mathrm{CKM} \text { unitarity } \Rightarrow & \left|V_{d L 13}\right|<10^{-1}, \quad\left|V_{d L 23}\right|<10^{-1 / 2}, \\
\text { Scalar resonance } \Rightarrow & \left|z_{4}^{d b}\right|<1 \div 10^{-2}, \quad\left|z_{4}^{s b}\right|<1 \div 10^{-1 / 2}, \quad\left|z_{4}^{d s}\right|<10^{-4} \div 10^{-6}, \\
\text { Vector resonance } \Rightarrow & \left|V_{d L 33}^{*} V_{d L 31}\right|<10^{-1} \div 10^{-3}, \quad\left|V_{d L 33}^{*} V_{d L 32}\right|<1 \div 10^{-2} \\
& \left|V_{d L 32}^{*} V_{d L 31}\right|<10^{-3} \div 10^{-5}
\end{aligned}
$$

where we find convenient to define $z_{4}^{d_{\alpha} d_{\beta}}=V_{d L 3 \alpha}^{*} V_{d L 3 \beta} \sum_{\gamma \delta} V_{d R \gamma \beta} V_{d R \delta \alpha}^{*}$.

The range in the case of resonances is due to the unknown value of the masses and couplings of the resonances. The only constraints directly deriving from partial compositeness in the upsector are the ones from CKM unitarity: however, they require a quite mild hierarchy in the downsector mixing matrix, especially in the first generation. It should also be noted that the effect scales like $M_{*}^{-2}$, so increasing the mass of the top partners can help releasing the tension. The strongest constraints come from higher order operators (in the case of the $\mathrm{Z}$ boson FCNCs) and heavy resonances, thus their presence is more model dependent. Nevertheless, there is no way to avoid such contributions in general. A possible simple way to contemporarily fulfill all the limits is $V_{d L 13}=0$ and $\left|V_{d L 23}\right|<10^{-2}$, with $V_{d L 33}=O(1)$ and generic $V_{d R}$ : we do not regard this choice as particularly fine tuned; also other possibilities are available. A peculiar case is to have the down mass matrix hierarchical as in the up sector, forcing the unitary transformations to be

$$
V_{d L, R} \sim\left(\begin{array}{ccc}
O(1) & O(1) & O\left(\frac{m_{s}}{m_{b}}\right) \\
O(1) & O(1) & O\left(\frac{m_{s}}{m_{b}}\right) \\
O\left(\frac{m_{s}}{m_{b}}\right) & O\left(\frac{m_{s}}{m_{b}}\right) & 1
\end{array}\right) .
$$


This in general is not completely satisfactory because the constraints on the coefficients $C_{1}^{b d, s d}$ of down-type operators coming from the exchange of heavy vector resonances generate a residual tension, as they may be one order of magnitude larger than the bounds; however an agreement with experiments can be obtained by varying the mass and couplings of the resonances. The structure in Eq.(3.2) could be a consequence of $\lambda_{33}^{d} \gg \lambda_{\alpha \beta}^{d}$, such that $m_{D, 33} \simeq m_{b}$ while $m_{D, \alpha \beta} \simeq m_{s}$ for all the other entries. Notice that this changes all the coefficients discussed: we checked that this still satisfies all the experimental bounds. In the down sector mass terms all originate from the same operators and in principle no hierarchy is expected. A origin for such a hierarchy is by further extending the model and making the bottom partially composite, and fixing the other down masses to be of the order of the strange mass. This makes the down sector similar to the up sector, with a clear distinction between the $\{3,3\}$ entry and the others in the mass matrix and the form of the diagonalizing $V_{d L, d R}$ is a consequence. We also point out that the simultaneous holding of Eq.(2.11) and Eq.(3.2) for $V_{u L}$ and $V_{d L}$ respectively is in agreement with the observed values of the third family entries of the CKM matrix.

It is instructive to revisit the limits collected in Eq.(3.1) allowing the entries of $V_{d L}$ to be $O(1)$ complex numbers, apart from $\left|V_{d L 13}\right|<10^{-1}$ and $\left|V_{d L 23}\right|<10^{-1 / 2}$ because of CKM unitarity: this in turn implies $\left|V_{d L 31,32}\right| \lesssim 10^{-1 / 2}$ because of $V_{d L}$ unitarity. The masses of heavy resonances probed by reconsidering the processes discussed above under this view point span $4-50 \mathrm{TeV}$.

To conclude, we briefly address issues related to $\mathrm{CP}$ violation. So far we treated all parameters as real: the suppressions we find are actually enough also for the imaginary parts. However some flavour conserving $\mathrm{CP}$ violating processes such as the neutron EDM might be enhanced. The current experimental bound is [62] $\left|d_{n}\right|<2.9 \times 10^{-26} e \mathrm{~cm}$ at $90 \%$ CL. New physics effects can be sizable $[22,23,63]$ : we estimated the quarks EDMs, $d_{u, d}$, restricting to one loop diagrams with $Z, W$ and Higgs bosons and $\xi_{\uparrow}$ and $\xi_{\downarrow}$ fermions, obtaining $10^{-21} \div 10^{-24} \mathrm{e} \mathrm{cm}^{3}$. Special choices of parameters or unitary matrices $V_{d L, R}$ might reduce $d_{d}$. In the up sector there are some fixed contributions, coming from Higgs exchange, and we have to assume that additional cancellations are at work or that the relative phase between $V_{u L 31}^{*}$ and $V_{u R 31}$ is small, less than $10^{-4}$. A full understanding of the neutron EDM relies on a complete theory of flavour and it is outside the scope of our effective parametrization: for this reason we do not include it in our global analysis.

\section{Conclusions}

We explored the possibility that the top quark mass has a different origin than the masses of the other quarks (and leptons). This can arise in models of pNGB Higgs. Partial compositeness is responsible for generating top quark mass and the Higgs potential, and an unrelated source of mass is represented by deformations of the strong sector that generate bilinear couplings suppressed by a scale larger than the condensation scale. In order to avoid four-fermion contributions to FCNCs, one needs this scale to be above $10^{5} \mathrm{TeV}$, which is enough to generate the bottom, charm and tau masses, but not the top mass, if the dynamics is near-conformal down to the condensation scale: the contribution of partial compositeness is crucial to achieve a large enough top mass.

We showed that this scenario is compatible with bounds from precision measurements of the quark couplings and from flavour constraints, without the need to assume a flavour symmetry in the

\footnotetext{
${ }^{3}$ We neglect QCD running effects, expected to be $O(1)$.
} 
underlying dynamics for the up sector. Hence, while the top is naturally singled out as the heaviest of the SM quarks, the direct Yukawa couplings can be anarchic. This property is due to suppressions in the corrections of order $v^{2} / f^{2}$ in the top sector, and $\left(m_{c} v\right)^{2} /\left(m_{t} f\right)^{2}$ in the light quarks sector. The situation is different for the down quarks: in the case where partial compositeness is not employed for the bottom mass, we observe only corrections of order $v^{2} / f^{2}$, notably in the unitarity of the CKM matrix. Therefore one is forced to ask for a certain hierarchy (requiring some alignment) in the mixing in the down sector. However, allowing the bottom to be partially composite, eases the tension if the contribution of direct couplings is smaller than the bottom mass, for instance as large as the strange mass, and a fully anarchic scenario becomes plausible. In this case, the hierarchy in the mixing in the SM fermion sector is enough to suppress effects in the unitarity of the CKM matrix and anarchic direct couplings are fully allowed. We do not expect significant deviations in flavour processes and the estimated sensitivities for the next run of LHC are still above the expected top flavour violating decays rates. On the other hand the neutron EDM might need a dedicated study and could be a significant test bench.

A smoking gun of our model are heavy coloured fermions. A discovery of the vector-like top (and bottom) partners, along with the absence of light fermion partners at the LHC is a genuine prediction of our framework. Finally, we want to emphasize that, while we analysed in detail the minimal case of the coset $\mathrm{SO}(5) / \mathrm{SO}(4)$ with top partners belonging to a four-plet and singlet of $\mathrm{SO}(4)\left(\right.$ aka $\mathrm{MCHM}_{5}$ ), our results are quite general. We showed how they can be extended to cases where the top (and bottom) partners belong to larger representations of $\mathrm{SO}(4)$ and cases where the coset is larger. Our conclusions are therefore rather solid under variations of the models.

\section{Acknowledgements}

A.P. acknowledges the organizing committee of Planck 2015 for the successful conference. He also thanks all the participants for creating a stimulating and friendly atmosphere. A.P. is grateful for support to IBS Korea under system code IBS-R017-D1-2015-a00.

\section{References}

[1] G. Cacciapaglia, H. Cai, T. Flacke, S. J. Lee, A. Parolini and H. Serôdio, JHEP 1506 (2015) 085 [arXiv:1501.03818 [hep-ph]].

[2] D. B. Kaplan, Nucl. Phys. B 365 (1991) 259.

[3] S. Dimopoulos and L. Susskind, Nucl. Phys. B 155, 237 (1979).

[4] E. Eichten and K. D. Lane, Phys. Lett. B 90 (1980) 125.

[5] S. Dimopoulos and J. R. Ellis, Nucl. Phys. B 182 (1982) 505.

[6] L. Randall and R. Sundrum, Phys. Rev. Lett. 83, 3370 (1999) [arXiv:hep-ph/9905221].

[7] N. Arkani-Hamed, M. Porrati and L. Randall, JHEP 0108 (2001) 017 [arXiv:hep-th/0012148].

[8] R. Rattazzi and A. Zaffaroni, JHEP 0104 (2001) 021 [arXiv:hep-th/0012248].

[9] J. M. Maldacena, Int. J. Theor. Phys. 38, 1113 (1999) [Adv. Theor. Math. Phys. 2, 231 (1998)] [arXiv:hep-th/9711200]. 
[10] R. Contino, Y. Nomura and A. Pomarol, Nucl. Phys. B 671 (2003) 148 [arXiv:hep-ph/0306259].

[11] K. Agashe, R. Contino and A. Pomarol, Nucl. Phys. B 719 (2005) 165 [arXiv:hep-ph/0412089].

[12] B. Bellazzini, C. Csàki and J. Serra, Eur. Phys. J. C 74 (2014) 5, 2766 [arXiv:1401.2457 [hep-ph]].

[13] G. Panico and A. Wulzer, arXiv:1506.01961 [hep-ph].

[14] N. Arkani-Hamed and M. Schmaltz, Phys. Rev. D 61 (2000) 033005 [arXiv:hep-ph/9903417].

[15] J. Galloway, J. A. Evans, M. A. Luty and R. A. Tacchi, JHEP 1010 (2010) 086 [arXiv:1001.1361 [hep-ph]].

[16] F. Caracciolo, A. Parolini and M. Serone, JHEP 1302 (2013) 066 [arXiv:1211.7290 [hep-ph]].

[17] J. Barnard, T. Gherghetta and T. S. Ray, JHEP 1402 (2014) 002 [arXiv:1311.6562 [hep-ph]].

[18] G. Ferretti and D. Karateev, JHEP 1403 (2014) 077 [arXiv:1312.5330 [hep-ph]].

[19] D. Marzocca, A. Parolini and M. Serone, JHEP 1403 (2014) 099 [arXiv:1312.5664 [hep-ph]].

[20] G. Ferretti, JHEP 1406 (2014) 142 [arXiv:1404.7137 [hep-ph]].

[21] A. Parolini, Phys. Rev. D 90 (2014) 11, 115026 [arXiv:1405.4875 [hep-ph]].

[22] M. Redi and A. Weiler, JHEP 1111 (2011) 108 [arXiv:1106.6357 [hep-ph]].

[23] K. Agashe, G. Perez and A. Soni, Phys. Rev. D 71, 016002 (2005) [arXiv:hep-ph/0408134].

[24] C. Delaunay, O. Gedalia, S. J. Lee, G. Perez and E. Ponton, Phys. Rev. D 83, 115003 (2011) [arXiv:1007.0243 [hep-ph]].

[25] A. L. Fitzpatrick, G. Perez and L. Randall, Phys. Rev. Lett. 100, 171604 (2008) [arXiv:0710.1869 [hep-ph]].

[26] G. Cacciapaglia, C. Csaki, J. Galloway, G. Marandella, J. Terning and A. Weiler, JHEP 0804, 006 (2008) [arXiv:0709.1714 [hep-ph]].

[27] C. Csaki, A. Falkowski and A. Weiler, Phys. Rev. D 80, 016001 (2009) [arXiv:0806.3757 [hep-ph]].

[28] J. Santiago, JHEP 0812, 046 (2008) [arXiv:0806.1230 [hep-ph]].

[29] M. C. Chen and H. B. Yu, Phys. Lett. B 672, 253 (2009) [arXiv:0804.2503 [hep-ph]].

[30] C. Csaki, G. Perez, Z. Surujon and A. Weiler, Phys. Rev. D 81, 075025 (2010) [arXiv:0907.0474 [hep-ph]].

[31] C. Csaki, A. Falkowski and A. Weiler, JHEP 0809, 008 (2008) [arXiv:0804.1954 [hep-ph]].

[32] R. Barbieri, G. Isidori, J. Jones-Perez, P. Lodone and D. M. Straub, Eur. Phys. J. C 71 (2011) 1725 [arXiv:1105.2296 [hep-ph]].

[33] R. Barbieri, D. Buttazzo, F. Sala and D. M. Straub, JHEP 1207 (2012) 181 [arXiv:1203.4218 [hep-ph]].

[34] M. A. Luty and T. Okui, JHEP 0609 (2006) 070 [arXiv:hep-ph/0409274].

[35] N. Craig, D. Green and A. Katz, JHEP 1107 (2011) 045 [arXiv:1103.3708 [hep-ph]].

[36] W. Altmannshofer, S. Gori, A. L. Kagan, L. Silvestrini and J. Zupan, arXiv:1507.07927 [hep-ph].

[37] D. Ghosh, R. S. Gupta and G. Perez, arXiv:1508.01501 [hep-ph].

[38] K. Agashe, R. Contino, L. Da Rold and A. Pomarol, Phys. Lett. B 641 (2006) 62 [arXiv:hep-ph/0605341]. 
[39] R. Contino, L. Da Rold and A. Pomarol, Phys. Rev. D 75 (2007) 055014 [arXiv:hep-ph/0612048].

[40] K. Agashe and R. Contino, Nucl. Phys. B 742 (2006) 59 [arXiv:hep-ph/0510164].

[41] A. De Simone, O. Matsedonskyi, R. Rattazzi and A. Wulzer, JHEP 1304 (2013) 004 [arXiv:1211.5663 [hep-ph]].

[42] S. Chatrchyan et al. [CMS Collaboration], Phys. Lett. B 729 (2014) 149 [arXiv:1311.7667 [hep-ex]].

[43] S. Chatrchyan et al. [CMS Collaboration], Phys. Rev. Lett. 112 (2014) 171801 [arXiv:1312.2391 [hep-ex]].

[44] G. Aad et al. [ATLAS Collaboration], [arXiv:1505.04306 [hep-ex]].

[45] O. Matsedonskyi, G. Panico and A. Wulzer, JHEP 1301 (2013) 164 [arXiv:1204.6333 [hep-ph]].

[46] D. Marzocca, M. Serone and J. Shu, JHEP 1208 (2012) 013 [arXiv:1205.0770 [hep-ph]].

[47] A. Pomarol and F. Riva, JHEP 1208 (2012) 135 [arXiv:1205.6434 [hep-ph]].

[48] O. Matsedonskyi, [arXiv:1411.4638 [hep-ph]].

[49] N. Vignaroli, Phys. Rev. D 86 (2012) 115011 [arXiv:1204.0478 [hep-ph]].

[50] C. Grojean, O. Matsedonskyi and G. Panico, JHEP 1310 (2013) 160 [arXiv:1306.4655 [hep-ph]].

[51] G. Aad et al. [ATLAS Collaboration], JHEP 1406 (2014) 008 [arXiv:1403.6293 [hep-ex]].

[52] S. Chatrchyan et al. [CMS Collaboration], Phys. Rev. Lett. 112 (2014) 171802 [arXiv:1312.4194 [hep-ex]].

[53] CMS Collaboration [CMS Collaboration], CMS-PAS-HIG-13-034.

[54] R. Rattazzi, V. S. Rychkov, E. Tonni and A. Vichi, JHEP 0812 (2008) 031 [arXiv:0807.0004 [hep-th]].

[55] F. Caracciolo, A. C. Echeverri, B. von Harling and M. Serone, JHEP 1410 (2014) 20 [arXiv:1406.7845 [hep-th]].

[56] G. Isidori, Y. Nir and G. Perez, Ann. Rev. Nucl. Part. Sci. 60 (2010) 355 [arXiv:1002.0900 [hep-ph]].

[57] L. Calibbi, Z. Lalak, S. Pokorski and R. Ziegler, JHEP 1207, 004 (2012) [arXiv:1204.1275 [hep-ph]].

[58] G. F. Giudice, C. Grojean, A. Pomarol and R. Rattazzi, JHEP 0706 (2007) 045 [arXiv:hep-ph/0703164].

[59] R. Barbieri, B. Bellazzini, V. S. Rychkov and A. Varagnolo, Phys. Rev. D 76 (2007) 115008 [arXiv:0706.0432 [hep-ph]].

[60] R. Contino, arXiv:1005.4269 [hep-ph].

[61] P. P. Giardino, K. Kannike, I. Masina, M. Raidal and A. Strumia, JHEP 1405 (2014) 046 [arXiv:1303.3570 [hep-ph]].

[62] C. A. Baker, D. D. Doyle, P. Geltenbort, K. Green, M. G. D. van der Grinten, P. G. Harris, P. Iaydjiev and S. N. Ivanov et al., Phys. Rev. Lett. 97 (2006) 131801 [arXiv:hep-ex/0602020].

[63] M. König, M. Neubert and D. M. Straub, Eur. Phys. J. C 74 (2014) 7, 2945 [arXiv:1403.2756 [hep-ph]]. 